\title{
Research status and trends of downwash airflow of spray UAVs in agriculture
}

\author{
Shenghui Yang, Yongjun Zheng*, Xingxing Liu \\ (College of Engineering, China Agricultural University, Beijing, 100083, China)
}

\begin{abstract}
Downwash airflow of spray UAVs in agriculture has been gradually focused on and a few studies have already been involved in it. Thus, this paper firstly reviewed the current research of downwash airflow of UAVs for agricultural spray. It is found that Chinese scholars have published the majority of the studies in this area. Furthermore, two aspects of this kind of research, one, the work related to the caused results of UAV downwash acting on spray, the other, that regarding downwash itself for investigation, were analysed. It is illustrated that the present research usually uses water-sensitive paper, electronic devices, like wind-speed arrays and PIV, and CFD simulation. However, the existed electronic devices have some limitations, such as lacking of the ability of showing and demonstration of downwash in big enough areas in detail during recording. Moreover, the studies directly related to downwash have been just launched around the world and the examination for multi-rotor UAVs is even seriously not enough. Therefore, in terms of research trends, it is significant to develop specialised sensors for downwash measurement. The general and reliable CFD models of a class of UAVs are also needed. In addition, the combination of UAV downwash with real applications and actual production is important for the clarification of the details in different situations.

Keywords: spray UAV, downwash, representation results, investigation methods
\end{abstract}

DOI: $10.33440 /$ j.ijpaa.20190201.0023

Citation: Yang S H, Zheng Y J, Liu X X. Research status and trends of downwash airflow of spray UAVs in agriculture. Int J Precis Agric Aviat, 2019; 2(1): 1-8.

\section{Introduction}

At present, the approach of control of both pests and disease in agriculture is pesticide spraying. With the development of technology, unmanned aerial vehicles (UAVs), including fixed-wing airplanes and rotor aircraft, have been gradually utilised for spray due to their high effectiveness and great efficiency ${ }^{[1-6]}$. During spraying, the downwash airflow passing by aircraft or through rotors can form a strong flow field to plants, able to further atomise droplets for better deposition onto crop surface. In addition, this kind of airflow can restrict and then deliver droplets into canopies to ensure a significant penetration effect. Therefore, the spray airflow has positive relations on spray swath, deposition and drift, determining the effect of operations ${ }^{[7]}$. It is necessary to investigate and clarify the distribution law of downwash.

This paper firstly reviewed the existed research of the downwash of agricultural UAVs and then proposed research trends based on the current limitations of these studies.

\section{Research progress of downwash airflow of the UAVs for spray}

\subsection{Overview of current research}

Figure 1 shows the proportions of current papers focusing on different topics about downwash airflow of agricultural spray UAVs. 53 academic published papers could be searched. According to Figure 1a, most of the studies regarding UAVs were completed by Chinese. Only several international scholars have

Received date: 2019-10-18 Accepted date: 2019-10-20

Biographies: Shenghui YANG, PhD, research interests: computer measurement and control, precision agricultural aviation and spray, Email: yshgxy@cau.edu.cn

* Corresponding author: Yongjun Zheng, PhD, Professor, research interests: computer measurement and control, applied technology of agricultural aviation. College of Engineering, China Agricultural University, Beijing, 100083, China. Tel: +86-10-62736385, Email: zyj@cau.edu.cn. paid attention to either spraying or downwash of UAVs, in terms of agricultural spray drones.

Besides, operation factors, such as flight height, flight speed and UAV type, could affect downwash, and then the downwash is able to impact the distribution or penetration of droplets based on these variations due to the strongly positive relations between wind velocity and droplets ${ }^{[8]}$. Thus, the relation between operation factors and droplet distributions is the caused result of downwash acting on spray, belonging to an indirect characterization of downwash. Generally, water-sensitive paper was used in these studies, but the main purpose was for the collection of droplets to evaluate the change of spray factors rather than the analysis of the relation between downwash and spray. Thus, these studies were not counted into the examination methods of downwash. According to Figure 1b, about half of these studies concentrated on this indirect relation, while more than a half research focused on the downwash itself and its examination methods.

In terms of examination methods, from Figure 1c, about 55 percent of the studies focused on the utilisation of Computational Fluid Dynamics (CFD), while approximately 44 percent of the research was mainly related to using materials or electronic devices. Furthermore, single-rotor downwash was the majority of the CFD studies (Figure 1d). As for materials and devices, wind-speed array, water-sensitive paper, camera and Particle Image Velocimetry (PIV) are the four commonly used approaches, while the first one was generally used by researchers (Figure 1e).

2.2 Key technology of caused results of UAV downwash acting on spray

It is proposed that the distribution of droplets is the presentation and performance of UAV downwash since the variation of spray factors could impact the intensity of downwash, in spite of an indirect relation.

Figure 2a shows the percentage of nationality of the current research on this key technology. China is the main region where the authors of this research were. Moreover, Figure 2b shows the 
year that this research was published. Year 2016 is a key point of the increase of this research. Moreover, according to Figure 2c, the commonly used method to investigate downwash is Computational Fluid Dynamics (CFD).

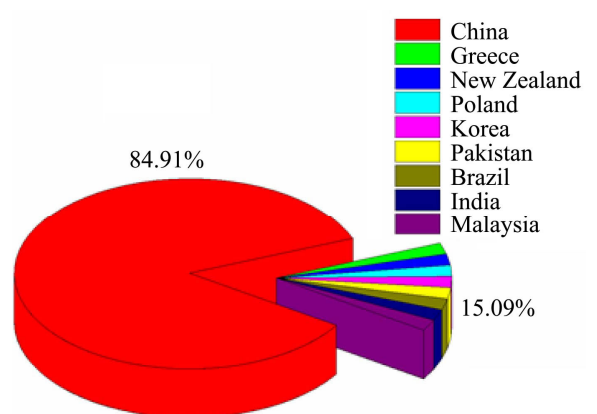

a. The nationality of the published relevant studies

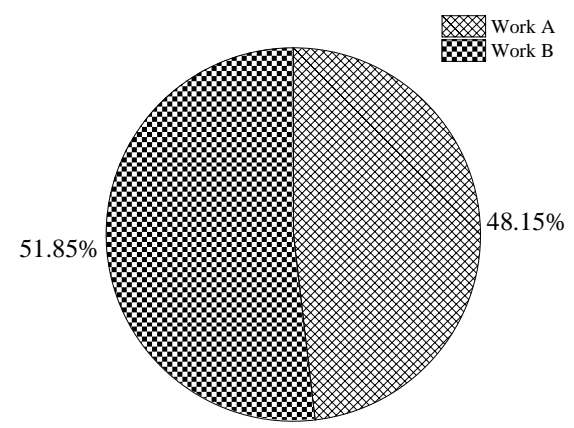

Work A: the work related to the caused result of downwash acting on spray, such as operation factors and spraying pressure; Work B: the work directly related to downwash and its examination.

b. The current studies related to downwash

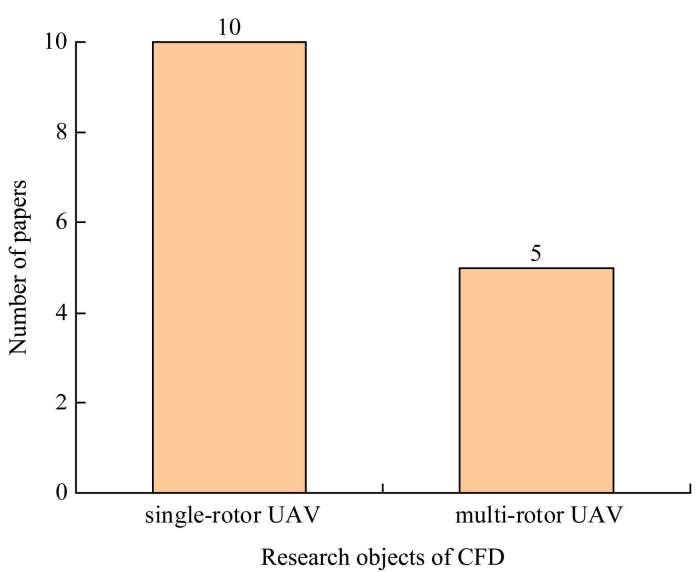

d. The research aspects and papers regarding the CFD simulation of downwash of spray UAVs

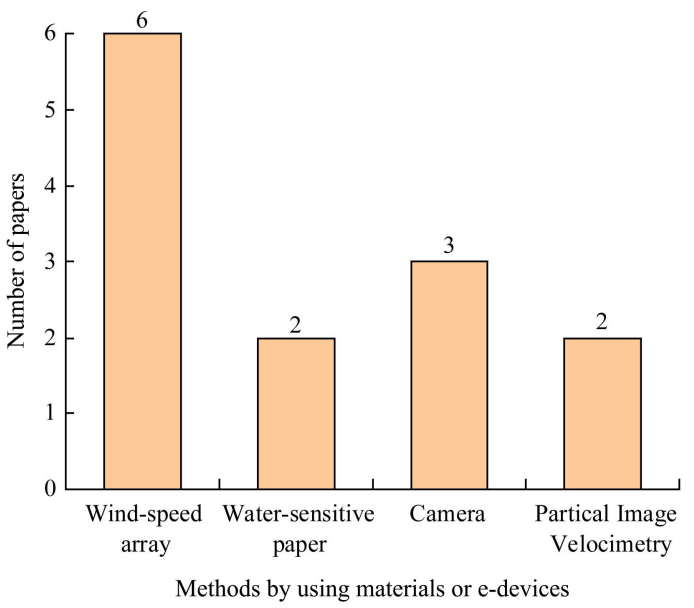

e. The research aspects and papers regarding the examination methods by using materials or electronic devices

Figure 1 The overview of the current status of the studies, showing the research points being concentrated on and the nationality of the relevant academic papers throughout the world

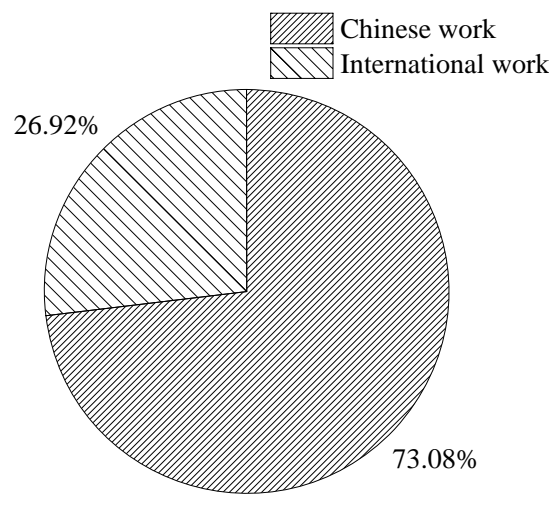

a. The nationality of the studies on the representation results of UAV downwash affecting spray

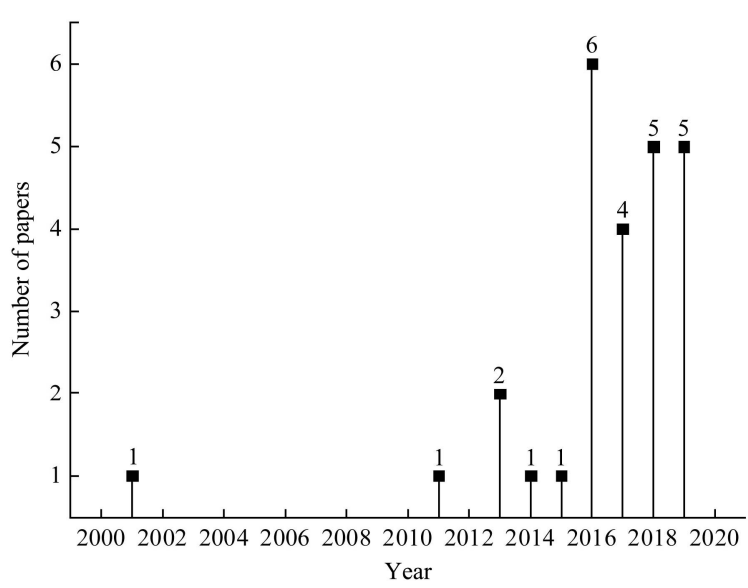

b. The year of the studies on the representation results of UAV downwash affecting spray

Figure 2 Current status of the studies on the representation results of UAV downwash affecting spray, indicating that first, these studies were completed by Chinese researchers and second, mainly focused on from 2016

A lot of studies have been involved in downwash characterization since 2013 in China. Scholars like $\mathrm{Gao}^{[9]}$ and Yang $^{[10]}$ started to focus on the effect from flight parameters, including heights and velocities, on droplet distributions. Several types of UAVs were analysed, such as Af-811 and WSZ-2410, and the optimal parameter pairs of operations were found for wheat and maize, while Xun paid attention on operation parameters, wind speed, spray pressure and pesticide for pest control of rice ${ }^{[11]}$. These three detailed and fundamental studies launched a rapid development of UAVs in China and attracted many interests. Then, the number of this kind of study highly increased from 2016 as well as expanded to other sprayed targets. For instance, Zhang 
et al started to focus on UAV application for citrus and found the variation of droplet distribution ${ }^{[12]}$, while Tang et al concentrated on the deposition changes affected by tree shapes ${ }^{[13]}$. Similar studies regarding UAVs and spray factors include T10 UAV for wheat scab control ${ }^{[14]}$, Response Surface Method for the test of P20 UAV parameters ${ }^{[15]}$, tree shapes and flight heights for the deposition of 3W-LWS-Q60S ${ }^{[16]}$, the comparison of four types of $\mathrm{UAVs}^{[17]}$, the method of detection of effective spray swath of 3WQF120-12 $2^{[18]}$, different operation parameters for several stages of corns ${ }^{[19]}$, low-attitude droplets deposition ${ }^{[20]}$, drift caused by different types of UAVs ${ }^{[21]}$, deposition on pineapple trees impacted by operation parameters ${ }^{[22]}$ and the control effect of wheat powdery mildew using different operation parameters ${ }^{[23]}$. Until 2019, this kind of study is still heated discussed ${ }^{[24-26]}$. All the research in China mentioned above supported great improvements of spraying technology of UAVs in Chinese agricultural aviation, offering an indirect relation to downwash.

International studies on aerial spray were launched earlier. In 2001, Greek scholars have started to compare the efficacy of several products used for spray ${ }^{[27]}$ and then Gaskin et al investigated the droplet distribution of Squirrel AS350 BA helicopter on fruit trees ${ }^{[28]}$. Nonetheless, only a little international research has been progressed to be related to the caused results of UAV downwash until now. Berner et al from Portland testified the relation between UAV rotor speed and spray deposition. The
UAV, DJI S 900, was moved along a track with a fixed height and a constant spraying pressure. Three rotor speeds, $0 \mathrm{r} \cdot \mathrm{min}^{-1}$, $1500 \mathrm{r} \cdot \mathrm{min}^{-1}$ and $4000 \mathrm{r} \cdot \mathrm{min}^{-1}$ were tested ${ }^{[29]}$. Furthermore, in 2018, Korean researchers examined downward wind speeds, spraying widths, falling particle quantities and particle sizes based on water-sensitive paper. Three heights and two speeds of a six-rotor UAV were set. Results indicated that $3 \mathrm{~m}$ height with $3 \mathrm{~m} \cdot \mathrm{s}^{-1}$ was the superior choice of spraying for Chinese cabbage ${ }^{[30]}$. In addition, there is only one study left that is relevant to this topic. Hussain et al from Pakistan justified the optimal operation parameters of a six-rotor UAV, which is the latest achievement of international research on UAV spray ${ }^{[31]}$. Other studies from Brazil $^{[32]}$ and India ${ }^{[33]}$ also paid attention to UAV spray but were mainly referred to route planning during spraying. Based on the international papers mentioned above, on one hand, the scholars discussing the effect from operation parameters of UAVs or rotor numbers on spray are much less than the Chinese. On the other hand, these papers were fully justified in detail, having a good value of referring.

Figure 3 gives an example of the research on the caused results of UAV downwash acting on spray. An UAV was used, flying with different heights and over the trees with different shapes. Water-sensitive paper was exploited to acquire the droplets data for further evaluation of the deposition changes of these conditions.

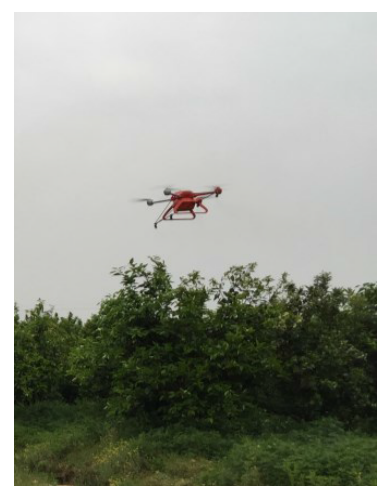

a 3W-LWS-Q60S UAV during operation

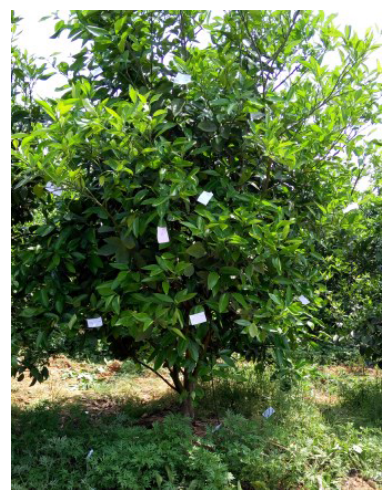

b the setting of water-sensitive pape

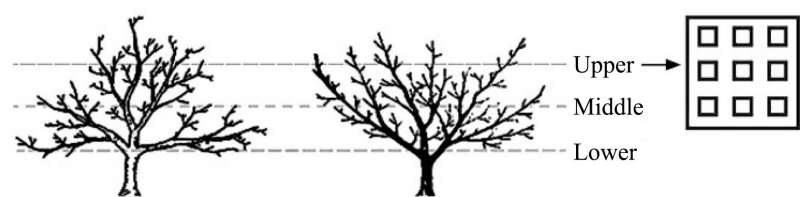

c different tree shapes

Figure 3 A typical instance of the caused results of UAV downwash acting on spray, showing that in this research, a four-rotor UAV was used to spray droplets and water-sensitive paper was set for collecting the droplets based on the variation of tree shapes and flight heights ${ }^{[12]}$

Both Chinese and international studies investigated droplet movement impacted by UAV factors, showing close thinking. However, it should be pointed out that droplet displacement and penetration is just the performance of UAV downwash acting on droplets, which means that it may not be the core issue to only focus on droplet behaviours.

\subsection{Key technology of examination methods of UAV downwash}

The essential question of droplets carried by downwash is the internal variation of downwash-itself. Hence, it is primary to illustrate how downwash distributes and what affects the distribution.

According to Figure 1a, compared with the studies on downwash characterization, although the research directly related to UAV downwash and its verification is more than a half, it is still insufficient as only 28 papers were available. Figure 4 shows the current status of these studies, indicating that Chinese studies are the majority and Computational Fluid Dynamics (CFD) is commonly utilised for the investigation of downwash (Figure 2c).

The earliest paper that could be searched in agriculture is from 2013. Xue conducted the research of the effect from aerial spray on rice ${ }^{[34]}$. This is an original work regarding downwash and gives a foundation and reference for future studies, since most of the later research applied the same approach.

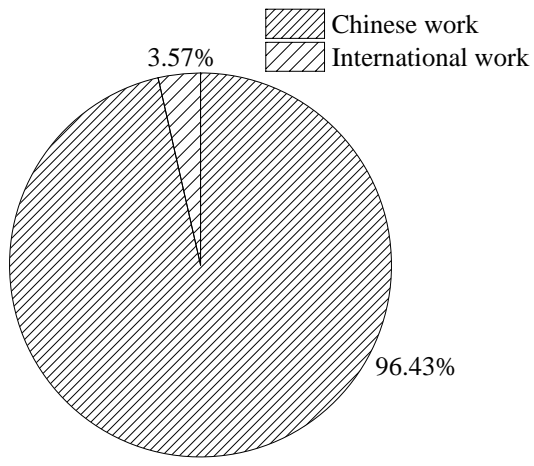

Figure 4 Current studies on the examination methods of UAV downwash

2.3.1 Examination by using materials or electronic devices

The electronic devices used for examination include wind-speed arrays, Particle Image Velocimetry (PIV) and cameras. 
Sometimes water-sensitive paper was employed. For using wind-speed arrays, in 2015, Li et al began to involve in this area. Figure 5 shows the scheme of the experiment. They exploited wind-speed arrays to investigate the distribution of downwash in order to help pollination by a type of UAV, SUMA18. Results illustrated that the distribution width is $Y$ direction $>X$ direction $>$ $Z$ direction. With the use of mathematical models like Gaussian Distribution, the downwash model was fitted ${ }^{[35]}$. Furthermore, they analysed the relation between downwash and pollen distribution by using a wireless measurement system for downwash. A consistent conclusion was made ${ }^{[36]}$. Their latest research was about using wind-speed arrays to measure the distribution and the speed of downwash airflow ${ }^{[37]}$. These are continuous studies on one kind of UAV and the conclusions are more and more in-depth, while Wang utilised a wind measurement system as well as a high-speed camera to find the wind distribution. Combined with wind-tunnel and real tests, the models between flight speeds and pollen distributions were obtained ${ }^{[38]}$. The approach began to be expanded. Chen et al also used a measurement system to detect the distribution of downwash of a multi-rotor UAV. They found that for M234-AT, Z-direction airflow had positive relations on deposition and drift $^{[39]}$. These are important works related to downwash. The studies by Li et al, Wang et al and Chen et al are mainly about the method of investigation but have the limitation on the clarification of the details of internal variation of downwash.

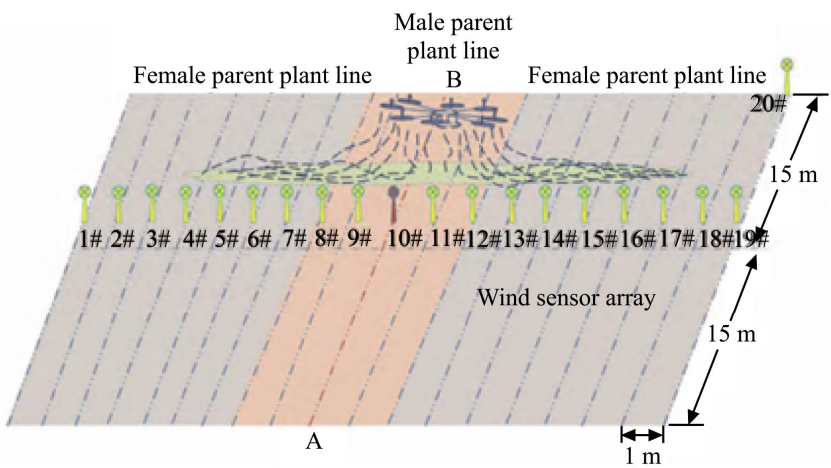

Figure 5 The downwash examination using wind-speed arrays, showing a typical setting of wind sensors to measure the distribution of airflow ${ }^{[35]}$

From the perspective of the method mainly using water-sensitive paper, Wang et al proposed and then optimised the method of spatial quality balance of droplets to examine the downwash of a single-rotor UAV, 3WQF80-10. As shown in Figure 6, water-sensitive paper was used in their research and they found that the wind speed in $\mathrm{Y}$ direction was the largest ${ }^{[40,41]}$. Their conclusion was similar to that by $\mathrm{Li}$ et al, provided with a good value of reference, but not able to specify the details and variation process of downwash.

With the improvement of hardware, Particle Image Velocimetry (PIV) was applied in the study by Tang et al to test the droplets distribution impacted by eight rotors in details. Figure 7 offers a typical instance of PIV testing. One nozzle was used and it was found that the downwash velocity was able to not only change the deposition zone of the droplets, but also influence their distribution $^{[42]}$. This is the first paper using PIV to observe the detail of droplet movement in agricultural aviation. However, it did not demonstrate the essential impact from wind changes on droplet motion and did test one nozzle only. A similar study was carried out by Luo in 2018, utilising a developed platform to test one-nozzle distribution affected by downwind ${ }^{[43]}$. Nevertheless, it had the same limitation.

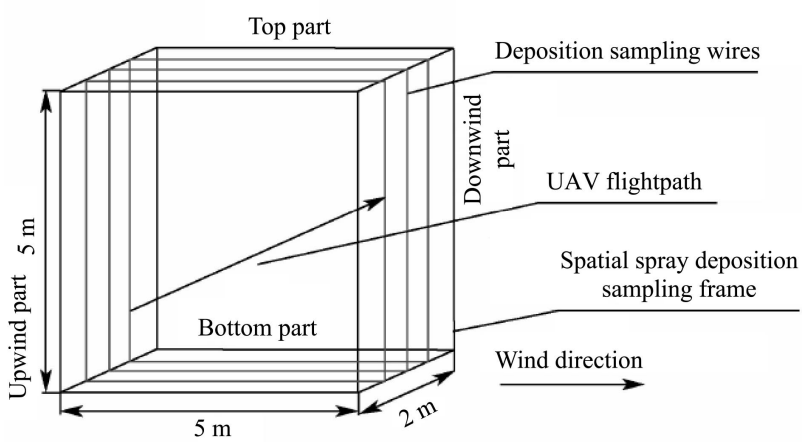

Figure 6 The testing method of spatial pesticide spraying deposition quality balance, proposed by Wang et al to collect droplets by water-sensitive paper and then do backstepping to evaluate the downwash distribution of three kinds of UAVs based on the droplets deposition in different layers ${ }^{[40]}$

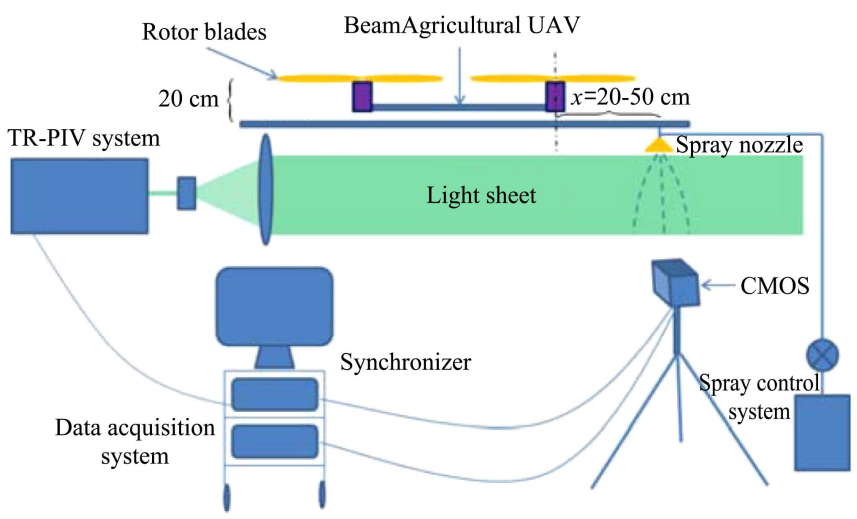

Figure 7 PIV for testing the distribution of droplets affected by downwash $^{[42]}$

Other devices, such as camera and the UAVs for photograph, were utilised, as shown in Figure 8. The group including $\mathrm{Li}$ and Guo from South China Agricultural University has been concentrated on vortex structure. They used the ratio between the maximum UAV size and the length of a side caused by downwash onto plants to represent the vortex size. An UAV for camera was exploited beyond the spraying UAV to take pictures of the shape by from downwash to plants. Then, the ratio was calculated and droplet distribution was analysed combined with this condition ${ }^{[44,45]}$ It is a novel idea, but different plants could be stressed and forced to form different shapes by downwash while the downwash intensity caused by the same UAV was equal in the same conditions. Therefore, using the ratio to represent the vortex scale needs further verifications.

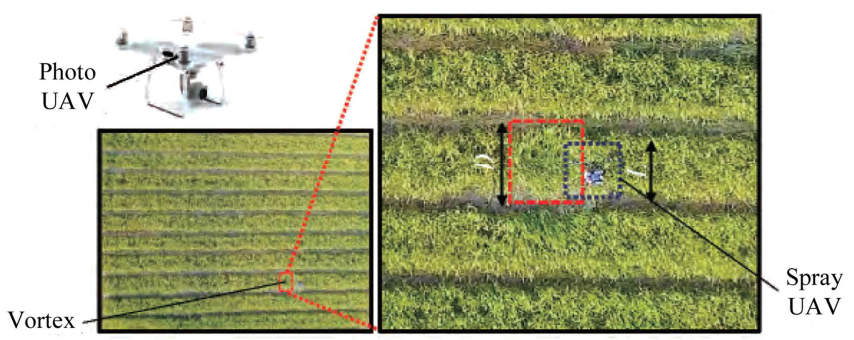

Figure 8 An UAV for photograph used for taking pictures of vortexes to calculate a vortex ratio proposed by the authors ${ }^{[44]}$

Based on this point mentioned above, the electronic devices currently used for downwash measurement and detection could not either give the details of the process of variation of downwash or 
capture enough big areas, which may limit the development of verification and investigation of downwash.

\subsubsection{Examination by using CFD}

As a novel and developing technology, CFD simulation has been commonly used since 2015 because of its controllable accuracy, great intuition and good details ${ }^{[46]}$. In agricultural aviation, scholars often use CFD to demonstrate the distribution of downwash. Although some of them combined sensors or water-sensitive paper, CFD was the major means. The equipment or materials used were just tools for verification, no longer fundamental ways of specifications of downwash.

In terms of single-rotor UAV, Xue firstly utilised CFD to simulate the distribution of UAV downwash and then verified the CFD models via wind-speed arrays. Combined with the factors from the UAV and its spray system, the prediction models of drift and deposition were developed ${ }^{[33]}$. Then, Zhang et al conducted a two-phase CFD simulation of single-rotor downwash and pesticide droplets in 2015. The results were compared with those of polyester cards ${ }^{[47]}$. This is the second earliest work on the downwash of unmanned helicopters, but the errors of the comparative experiments were big, so further discussion may be requested. Shi et al also published an article in 2015 to analyse the CFD models of downwash of an unmanned helicopter and obtained the characteristics of downwash distribution ${ }^{[48]}$. Subsequently, they added discrete phase to simulate droplet distribution within downwash ${ }^{[49]}$. Nonetheless, their studies contained CFD only, without verifications. Zamri et al conducted a similar study by CFD to analyse droplet movement but still lacked verifications ${ }^{[50]}$. As single-rotor UAVs or helicopters have only one propeller and may be relatively simple to complete simulation, the CFD research about them was increased from 2017. Zhang et al used CFD simulation combined with real hovering trials to obtain the characteristics of droplet distribution of $\mathrm{N}-3^{[51]}$. Yang et al simulated the distribution model of the rotor of $\mathrm{XV}-2$. Compared with experiments, they found how the airflow influenced the spray swath of the UAV and indicated that the CFD model was basically consistent with the experimental results ${ }^{[52]}$. Moreover, Wen et al mainly applied CFD to investigate the effect from wing-tip vortexes on droplets. Different flight speeds were simulated and the proportions between vortexes and drift were clarified $^{[53]}$. Xu very specifically analysed the flow and speed characteristics of a large-load unmanned helicopter. The velocity distribution of both flight and hovering states in different canopy heights were simulated and tested ${ }^{[54]}$. This is a comprehensive study on single-rotor downwash due to its combination of multiple factors. A similar study to $\mathrm{Xu}$ was conducted by Cao. The author was also from Jiangsu University and close methods were used $^{[55]}$. All the studies mentioned above could gave a technical reference for multi-rotor UAVs, but the difficulty of both simulation and calculation may be lower than multiple rotors.

For multi-rotor UAVs, the relevant research that can be searched is just from 2017. Two institutes are leading. The group including Yang and Xue from Nanjing Research Institute for Agricultural Mechanization continuously focused on multi-rotor CFD. They firstly applied CFD to simulate a six-rotor UAV, SLK-5, and combined with wind-investigation experiments to obtain the characteristics of both maximum entire velocity and Z-direction wind ${ }^{[56]}$. However, the test error was big (less than or equal to $12 \%$ ) so that the experimental design and the details of CFD models were required to be optimised. Thus, their later study improved the accuracy of both the experimental proposal and the CFD models and the error was less than or equal to $9 \%$. Furthermore, in hovering state, the relations from droplet distribution to 'airflow inlet' and 'airflow outlet' were gained ${ }^{[57]}$. Another close simulation for the analysis of drift was conducted ${ }^{[58]}$. Their research is coherent with good justifications, able to offer significant samples for the investigation of multi-rotor downwash. In terms of China Agricultural University, the team containing Zheng and Yang simulated the downwash flowing field of a type of six-rotor UAV, JF 01-10. The features and characteristics of the downwash field were obtained ${ }^{[59]}$, but the computation cost was high and further validation is needed to examine the models. Then, they conducted a qualitative test to try to verify the distribution of an eight-rotor downwash. Tracer was mixed in the spray liquid ${ }^{[60]}$. Although the results should be further discussed, it is the first attempt to intuitively verify the downwash of a multi-rotor UAV, since multi-rotor UAVs for plant protection are generally large and no devices including PIV could directly show the details.

In addition, it is pointed out that CFD method has also been used for investigate the airflow of fixed-wing UAVs in agriculture. Although this kind of fixed-wing airflow does not belong to downwash, ground-effect and droplet distribution were analysed based on it, which is very close to the approach of downwash examination. One published work by Zhang from Northwestern Polytechnical University in China and Tang from National Research Centre of Intelligent Equipment for Agriculture investigated the distribution of wake vortexes of a monoplane, Thrush 510G, identifying that the starboard vortex existed longer than the port one ${ }^{[61]}$. Ultrasonic anemometers were employed to acquire the airflow velocity during the aircraft passing by. It is a good instance of analysis of fixed-wing UAVs. However, no more literature about the downwash of fixed-wing UAVs could be searched, indicating that the study in this area is extremely insufficient.

Figure 9 shows several examples of CFD models of downwash caused by rotors.
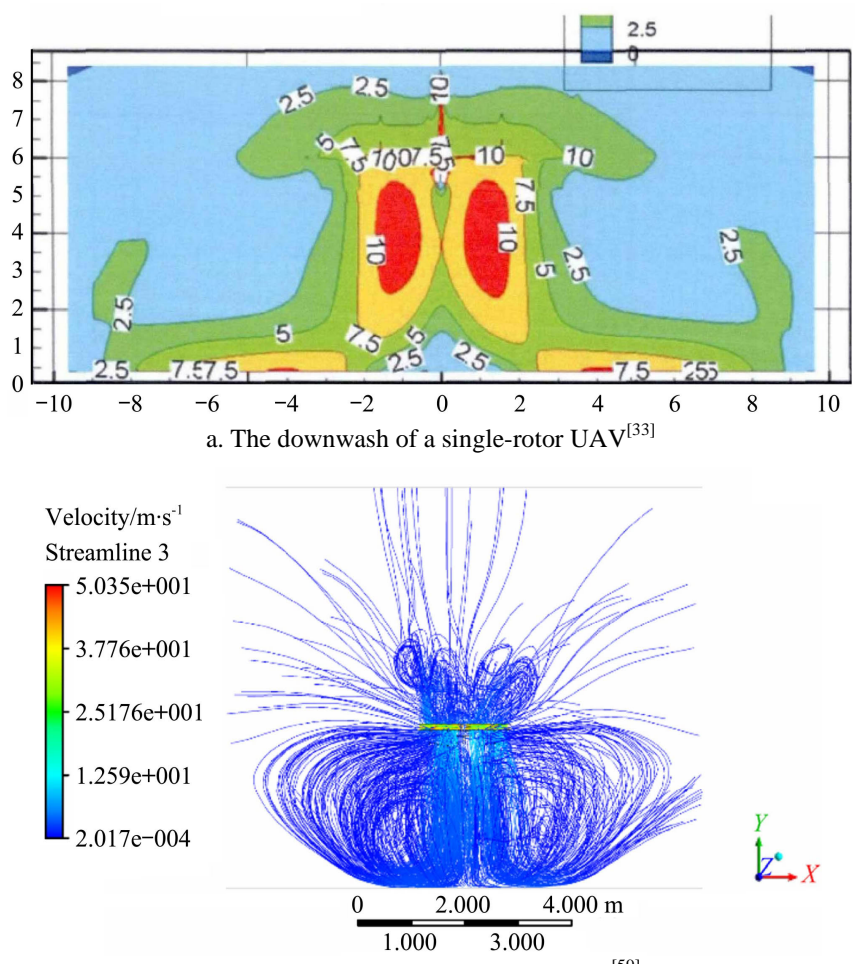

b. The downwash of a six-rotor $\mathrm{UAV}^{[59]}$

Figure 9 The examples of the CFD models of several types of UAVs 
3 Research trends of downwash airflow of the UAVs for spray

3.1 Development of specialised sensors for the measurement of downwash airflow

Although CFD simulation has been a commonly used approach, different meshing may have different accuracy ${ }^{[62]}$. Thus, the final step assisted by CFD models is still to examine and verify them. However, there are no suitable sensors specialised for downwash measurement to show the flowing and distributing of downwash in large scales in detail. Until know, wind-speed arrays and PIV have been exploited as the electronic devices for the detection of downwash, but they either are indirectly measurement that needs reverse calculation or measure within a very small section. Therefore, the specialised sensors, able to specify the transfer and diffusing of UAV downwash, should be developed.

\subsection{Development of general and reliable CFD models of downwash}

Current CFD simulation are established by setting several kinds of mathematical equation models, such as $k-\varepsilon$, Shear-Stress-Transfer (SST) and Large Eddy Simulation (LES). In spite of the similar results, each mathematical model has different preconditions. Considering meshing methods, the reliability of CFD models of a type of UAV has to be further discussed. Meanwhile, the existed CFD simulation is mainly referred for single-rotor UAVs, while there are many kinds of single or multiple rotor UAVs in the market. In other words, present CFD models are specifically developed for particular types of UAVs, not general for a class of UAVs. The UAVs having the same rotor numbers and similar sizes of both wheelbase and propellers should be considered together to analyse the similarity of their downwash. Thus, general and reliable CFD simulation should be established for a class of UAVs. Furthermore, the minimum requirements of from meshing to math model selection should be investigated.

\subsection{Discussion of the effect on different kinds of crops combined with application}

The ultimate purpose of downwash research is to improve the effectiveness and efficiency of pesticide spraying by UAVs. As a result, actual application of downwash is highly important. The target area and crop, such as paddy and maize, may be distinct, so different crops and trees should be specifically analysed with different conditions. For instance, the downwash penetration combined with droplets in canopies can be tested. Then, the common law of characteristics of distribution of downwash for a kind of plants can be integrated together as an achievement.

\subsection{Discussion of multi-UAV coordination combined with production}

Current studies on both spray effect and downwash are concentrated on single-UAV operation, while for large-area fields, it may be more actual and efficient to use several UAVs for operation. Consequently, if the downwash is clarified, its distribution should be combined to investigate what is the difference between one UAV and several UAVs and how the downwash can affect operation in several-UAV conditions. More laws and regular patterns need to be demonstrated, but there has been no beginning of multi-UAV coordination in agricultural spray.

\section{Conclusion}

This paper reviewed the current research status of downwash airflow of spray UAVs in agriculture. It was found that the research regarding operation factors, which was related to the representation results of UAV downwash affecting spray, had the heated discussion. In terms of downwash itself, the research focused on the investigation of downwash. Electronic devices, such as wind-speed arrays, PIV and camera, and CFD simulation were generally used. However, the studies directly related to downwash are just started throughout the world and those related to multi-rotor examination are insufficient, so it is really necessary to develop the specialised sensors for downwash measurement and the general and reliable CFD models of a class of UAVs. Meanwhile, the downwash research needs to be combined with real applications and actual production, to illustrate the laws for both different crops and multi-UAV operations.

\section{Acknowledgement}

This paper was supported and funded by National Key Research and Development Program of China, 2016YFD200700, and National Key Research and Development Program of China, 2017 YFD0701003.

\section{[References]}

[1] Tian Z W, Xue X Y, Li L, Cui L F, Wang G, Li Z J. Research status and prospects of spraying technology of plant-protection unmanned aerial vehicles. Journal of Chinese Agricultural Mechanization, 2019, 40(1): 37-45. (in Chinese with English Abstract) doi: 10.13733/j.jcam.issn. 2095-5553.2019.01.08

[2] He Y, Wu J J, Fang H, Zheng Q S, Xiao S P, Cen H Y. Research on deposition effect of droplets based on plant protection unmanned aerial vehicle: A review. Journal of Zhejiang University (Agric. \& Life Sci.), 2018, 44(4): 392-398. (in Chinese with English Abstract) doi: 10.13733/j.jcam.issn.2095-5553.2019.01.08

[3] Lan Y B, Chen S D, Fritz B K. Current status and future trends of precision agricultural aviation technologies. Int J Agric \& Biol Eng, 2017; 10(3): 1-17. doi: 10.3965/j.ijabe.20171003.3088

[4] Zhou Z Y, Ming R, Zang Y, He X G, Luo X W, Lan Y B. Development status and countermeasures of agricultural aviation in China. Transactions of the CSAE, 2017, 33(20): 1-13. (in Chinese with English Abstract) doi: 10.11975/j.issn.1002-6819.2017.20.001

[5] Zhang D Y, Lan Y B, Chen L P, Wang X, Liang D. Current status and future trends of agricultural aerial spraying technology in China. Transactions of the CSAM, 2014, 45(10): 53-59. (in Chinese with English Abstract) doi: 10.6041/j.issn.1000-1298.2014.10.009

[6] Zhou Z Y, Yuan W, Chen S D. Current status and future directions of rice plant protection machinery in China. Guangdong Agricultural Sciences, 2014, (5): 178-183. (in Chinese with English Abstract) doi: 10.3969/j.issn.1004-874X.2014.15.036

[7] Li J Y, Lan Y B, Shi Y Y. Research progress on airflow characteristics and field pesticide application system of rotary-wing UAV . Transactions of the CSAE, 2018, 34(12): 104-118. (in Chinese with English abstract) doi: 10.11975/j.issn.1002-6819.2018.12.013. http://www.tcsae.org

[8] An J L, Xiang W L, Han Z W, Xiao K T, Wang Z F, Wang X H, Wu J B, Yan $\mathrm{P} \mathrm{Z}$, Li J, Che Y, Li J, Li Y. Validation of the Institute of Atmospheric Physics emergency response model with the meteorological towers measurements and SF6 diffusion and pool fire experiments. Atmospheric Environment, 2013, 81: 60-67. doi: 10.1016/j.atmosenv. 2013.08.041

[9] Gao Y Y. Study on distribution of pesticide droplets in gramineous crop canopy and control effect sprayed by unmanned aerial vehicle. Master dissertation. Heilongjiang: Northeast Agricultural University, 2013: 1-44. (in Chinese with English Abstract)

[10] Yang S. Spray droplet deposition and distribution inside crop canopy and control efficiency applied by unmanned aerial vehicle. Master dissertation. Beijing: Chinese Academy of Agricultural Sciences, 2014: 1-44. (in Chinese with English Abstract)

[11] Xun D. Study on flight control parameters and efficiency for rice pest control by TH-80-1 plant protection UAV. Master dissertation. Hunan: Hunan Agricultural University, 2015:1-43. (in Chinese with English 
abstract)

[12] Zhang P, Lv Q, Yi S L, Liu Y, He S L, Xie R J, Zheng Y Q, Pan H Y, Deng L. Evaluation of spraying effect using small unmanned aerial vehicle (UAV) in citrus orchard. Journal of Fruit Science, 2016, 33(1): 34-42. (in Chinese with English abstract) doi: 10.13925/j.cnki.gsxb. 20150182

[13] Tang Y, Hou C J, Luo S M, Lin J T, Yang Z, Huang W F. Effects of operation height and tree shape on droplet deposition in citrus trees using an unmanned aerial vehicle. Computers and Electronics in Agriculture, 2018, 148: 1-7. doi: 10.1016/j.compag.2018.02.026

[14] Wang M. Study on technology parameters of T-10 type four rotor UAV and control effect on wheat scab. Master dissertation. Anhui: Anhui Agricultural University, 2016:1-43. (in Chinese with English Abstract)

[15] Qin W C, Xue X Y, Zhang S C, Gu W, Chen C. Optimization and test of spraying parameters for P20 multi-rotor electric unmanned aerial vehicle based on response surface method. Journal of Jiangsu University (Natural Science Edition), 2016, 37(5): 548-555. (in Chinese with English Abstract) doi: 10.3969/j.issn.1671-7775.2016.05.010

[16] Zhang P, Deng L, Lyu Q, He S L, Yi S L, Liu Y D, Yu Y X, Pan H Y. Effects of citrus tree-shape and spraying height of small unmanned aerial vehicle on droplet distribution. Int J Agric \& Biol Eng, 2016; 9(4): 45-52. doi: 10.3965/j.ijabe.20160904.2178

[17] Wang S L, Song J L, He X K, Song L, Wang X N, Wang C L, Wang Z C, Ling Y. Performances evaluation of four typical unmanned aerial vehicles used for pesticide application in China. Int J Agric \& Biol Eng, 2017; 10(4): 22-31. doi: 10.25165/j.ijabe.20171004.3219

[18] Chen S D, Lan Y B, Li J Y, Xu X J, Wang Z G, Peng B. Evaluation and test of effective spraying width of aerial spraying on plant protection UAV. Transactions of the CSAE, 2017, 33(7): 82-90. (in Chinese with English abstract). doi: 10.11975/j.issn.1002-6819.2017.07.011. http://www.tcsae.org

[19] Zheng Y J, Yang S H, Zhao C J, Chen L P, Lan Y B, Tan Y. Modelling operation parameters of UAV on spray effects at different growth stages of corns. Int J Agric \& Biol Eng, 2017, 10(3): 57-66. doi: 10.3965/j.ijabe.20171003.2578

[20] Wang L. Research on low-attitude droplets deposition and technology of variable spraying measurement and control system based on multi-rotor crop protection UAV. PhD dissertation. Beijing: China Agricultural University, 2017: 1-88. (in Chinese with English abstract)

[21] Wang X N, He X K, Song J L, Wang Z C, Wang C L, Wang S L, Wu R C, Meng Y H. Drift potential of UAV with adjuvants in aerial applications. Int J Agric \& Biol Eng, 2018; 11(5): 54-58. doi: 10.25165/j.ijabe. 20181105.3185

[22] Wang J, Lan Y B, Zhang H H, Zhang Y L, Wen S, Yao W X, Deng J J. Drift and deposition of pesticide applied by UAV on pineapple plants under different meteorological conditions. Int J Agric \& Biol Eng, 2018; 11(6): 5-12. doi: 10.25165/j.ijabe.20181106.4038

[23] Qin W C, Xue X Y, Zhang S M, Gu W, Wang B K. Droplet deposition and efficiency of fungicides sprayed with small UAV against wheat powdery mildew. Int J Agric \& Biol Eng, 2018; 11(2): 27-32. doi: 10.25165/j.ijabe.20181102.3157

[24] Hou C J, Tang Y, Luo S M, Lin J T, He Y, Zhuang J J, Huang W F. Optimization of control parameters of droplet density in citrus trees using UAVs and the Taguchi method. Int J Agric \& Biol Eng, 2019; 12(4): 1-9 doi: 10.25165/j.ijabe.20191204.4139

[25] Liao J, Zang Y, Luo X W, Zhou Z Y, Lan Y B, Zang Y, Gu X Y, Xu W M, Andrew J H. Optimization of variables for maximizing efficacy and efficiency in aerial spray application to cotton using unmanned aerial systems. Int J Agric \& Biol Eng, 2019; 12(2): 10-17. doi: 10.25165/ j.ijabe.20191202.4288

[26] Wang J, Lan Y B, Yao W X, Chen P C, Lin J L, Yan Y W. Effects of working height of single-rotor unmanned aerial vehicle on drift and droplets deposition distribution of areca tree. Transactions of the CSAM, 2019, 50(7): 109-119. (in Chinese with English abstract) doi:10.6041/ j.issn.1000-1298.2019.07.011

[27] Yee W L, Phillips P A, Faber B A. Effects of aerial spray volume, coverage, and sabadilla on Scirtothrips perseae (Thysanoptera: Thripidae). Journal of economic entomology, 2001, 94(5): 1085-1089. doi: 10.1603/0022-0493-94.5.1085

[28] Gaskin R E, Steele K D, Elliott G S. Concentrated lowvolume aerial sprays to improve spray distribution in large avocado trees. New Zealand Plant Protection, 2011, 64: 107-111. doi: 10.30843/nzpp.2011.64.5970

[29] Berner B, Chojnacki J. Use of drones in crop protection. 2017. doi: 10.24326/fmpmsa.2017.9
[30] Choi D S, Ma K C, Kim H J, Lee J H, Oh S A, Kim S G. Control Standards of Three Major Insect Pests of Chinese Cabbage (Brassica campestris) Using Drones for Pesticide Application. Korean journal of applied entomology, 2018, 57(4): 347-354. (in Korean with English abstract) doi: 10.5656/KSAE.2018.09.0.031

[31] Hussain S, Cheema M, Ahmad J M A, Latif M A, Ashraf S, Ahmad S. Spray Nidormity testing of unmanned aerial spraying system for precise agro-chemical applications. Pakistan Journal of Agricultural Sciences, 2019, 56(4). doi: 10.21162/PAKJAS/19.8594

[32] Bruno S F, Gustavo P, Geraldo P R F, Carvalho A C P L F, Gomes P H, Ueyama J. Fine-tuning of UAV control rules for spraying pesticides on crop fields: An Approach for dynamic environments. International Journal of Artificial Intelligence Tools, 2016, 25(1):1660003. doi: 10.1142/S0218213016600034

[33] Aditya S N, Prof S C K. Adoption and utilization of drones for advanced precision farming: A Review. International Journal on Recent and Innovation Trends in Computing and Communication, 2016, 4(5): 563-565. Available: https://ijritcc.org/index.php/ijritcc/article/view/2237. Access on [2016-05-31]

[34] Xue X Y. Applications of modern pesticide aerial application technology and the impact on rice quality. PhD dissertation. Jiangsu: Nanjing Agricultural University, 2013: 1-172. (in Chinese with English abstract)

[35] Li J Y, Zhou Z Y, Lan Y B, Hu L, Zang Y, Liu A M, Luo X W, Zhang T M. Distribution of canopy wind field produced by rotor unmanned aerial vehicle pollination operation. Transactions of the CSAE, 2015, 31(3): 77-86. (in Chinese with English abstract) doi: 10.3969/j.issn.1002-6819.2015. 03.011

[36] Li J Y, Lan Y B, Wang J W, Chen S D, Huang C, Liu Q, Liang Q P. Distribution law of rice pollen in the wind field of small UAV. Int J Agric \& Bio Eng, 2017; 10(4): 32-40. doi: 10.25165/j.ijabe.20171004.3103

[37] Li J Y, Shi Y J, Lan Y B, Guo S. Vertical distribution and vortex structure of rotor wind field under the influence of rice canopy. Computers and Electronics in Agriculture, 2019, 159: 140-146. doi: 10.1016/j.compag.2019.02.027

[38] Wang J W. Study on the Pollen Movement of Rice Based on the Wind Field of Unmanned Aerial Vehicle. Master Dissertation. Guangdong: South China Agricultural University, 2016: 1-55. (in Chinese with English abstract)

[39] Chen S D, Lan Y B, Bradley K F, Li J Y, Liu A M, Mao Y D. Effect of wind field below rotor on distribution of aerial spraying droplet deposition by using multi-rotor UAV. Transactions of the CSAM, 2017, 48(8): 105-113. (in Chinese with English abstract) doi: 10. 6041/j.issn.10001298. 2017.08. 011

[40] Wang C L, He X K, Wang X N, Wang Z C, Wang S L, Li L L, Jane B, Andreas H, Wang Z G, Mei S F. Distribution characteristics of pesticide application droplets deposition of unmanned aerial vehicle based on testing method of deposition quality balance. Transactions of the CSAE, 2016, 32(24): 89-97. (in Chinese with English abstract). doi: 10.11975/ j.issn.1002-6819.2016.24.012

[41] Wang C L, He X K, Wang X N, Wang Z C, Wang S L, Li L L, Jane B, Andreas H, Wang Z G. Testing method and distribution characteristics of spatial pesticide spraying deposition quality balance for unmanned aerial vehicle. Int J Agric \& Biol Eng, 2018; 11(2): 18-26. doi: 10.25165/j.ijabe.20181102.3187

[42] Tang Q, Zhang R R, Chen L P, Xu M, Yi T C, Zhang B. Droplets movement and deposition of an eight-rotor agricultural UAV in downwash flow field. Int J Agric \& Biol Eng, 2017; 10(3): 47-56. doi: 10.3965/j.ijabe.20171003.3075

[43] Luo B T. Research on Droplet Deposition Characteristics of Large-scale Plant Protection UAV Spraying Operation. Master dissertation. Jiangsu: Jiangsu University, 2018: 1-56. (in Chinese with English abstract)

[44] Li J Y, Guo S, Yao W X, Zhan Y L, Li Y F. Distribution Characteristics of Droplet Size in rice Field and Wind Tunnel Simulation Test under Airflow Operation. Transactions of the CSAM, 2019, 50(8): 148-156. (in Chinese with English abstract). doi: 10.6041/j.issn.1000-1298. 2019.08. 017

[45] Guo S, Li J Y, Yao W X, Zhan Y L, Li Y F, Shi Y Y. Distribution characteristics on droplet deposition of wind field vortex formed by multi-rotor UAV. PloS one, 2019, 14(7): e0220024. Available: https://doi.org/10.1371/journal.pone.0220024. Access on [2019-07-22]

[46] Zhang H X. Investigations on fidelity of high order accurate numerical simulation for computational fluid dynamics. ACTA Aerodynamica Sinica, 2016, 34(01): 1-4. (in Chinese with English abstract) doi: 
10.7638/kqdlxxb-2015.0211

[47] Zhang S C, Xue X Y, Qin W C, Sun Z, Ding S M, Zhou L X. Simulation and experimental verification of aerial spraying drift on Nf3 unmanned spraying helicopter. Transactions of the CSAE, 2015, 31(3): 87-93. (in Chinese) doi:10.3969/j.issn.1002-6819.2015.03.012

[48] Shi Q. Numerical simulation for downwash flow field of small-size unmanned helicopter hedgehopping. Journal of Drainage and Irrigation Machinery Engin, 2015, 33(6): 521-525. (in Chinese with English abstract) doi: 10.3969/j.issn.1674-8530.15.0073

[49] [49] Shi Q, Guan X P, Sun H W. Numerical simulation for spray field of small-size unmanned helicopter hedgehopping. Jiangsu Agricultural Sciences, 20-16, 44(9): 360-364. (in Chinese) doi: 10.15889 /j. issn. 1002-1302. 2016. 09. 103

[50] Zamri O, Kua Y Q, Sofian M, Nurhayati R. CFD simulation of aerial crop spraying[C]//OP Conference Series: Materials Science and Engineering. IOP Publishing, 2016, 160(1): 012028. doi: 10.1088/ 1757-899X/160/1/012028

[51] Zhang S C, Xue X Y, Sun Z, Zhou L X, Jin Y K. Downwash distribution of single-rotor unmanned agricultural helicopter on hovering state. Int $\mathrm{J}$ Agric \& Biol Eng, 2017; 10(5): 14-24. doi: 10.25165/j.ijabe.20171005. 3079

[52] Yang Z L, Ge L Z, Qi L J, Cheng Y F, Wu Y L. Influence of UAV rotor down-wash airflow on spray swath. Transactions of the CSAM, 2018, 49(01): 116-122. (in Chinese with English abstract) doi: 10.6041/j.issn.10001298.2018.01.014

[53] Wen S, Han J, Lan Y B, Yin X C, Lu Y H. Influence of wing tip vortex on drift of single rotor plant protection unmanned aerial vehicle. Transactions of the CSAM, 2018, 49(8): 127-137+160. (in Chinese with English abstract) doi:10.6041/j. issn. 1000-1298. 2018. 08. 015

[54] Xu W B. The Study of Flow and Sedimentary Characteristics during Spraying Process of Large-load Plant Protection Unmanned Helicopter. Master Dissertation. Jiangsu: Jiangsu University, 2017: 1-76. (in Chinese with English abstract)

[55] Cao S N. Numerical Analysis and Experimental Study on Spray Droplet Distribution of Single Rotor UAV. Master Dissertation. Jiangsu: Jiangsu University, 2019: 1-68. (in Chinese with English abstract)

[56] Yang F B, Xue X Y, Zhang L, Sun Z. Numerical simulation and experimental verification on downwash air flow of six-rotor agricultural unmanned aerial vehicle in hover. Int J Agric \& Biol Eng, 2017; 10(4): 41-53. doi: 10.25165/j.ijabe.20171004.3077

[57] Yang F B, Xue X Y, Cai C, Zhou Q Q. Effect of down wash airflow in hover on droplet motion law for multi-rotor unmanned plant protection machine. Transactions of the CSAE, 2018, 34(2): 64-73. (in Chinese with English abstract) doi: 10.11975/j.issn.1002-6819.2018.02.009

[58] Yang F B, Xue X Y, Cai C, Zhu S, Zhou Q Q. Numerical Simulation and analysis on spray drift movement of multirotor plant protection unmanned aerial vehicle. Energies, 2018, 11(9): 2399. Available: https://doi.org/ 10.3390/en11092399. Access on [2019-09-18]

[59] Zheng Y J, Yang S H, Liu X X, Wang J, Tomas N, Chen J, Tan Y. The computational fluid dynamic modeling of downwash flow field for a six-rotor UAV. Frontiers of Agricultural Science and Engineering, 2018, 5(02): 159-167. Available: https://doi.org/10.15302/J-FASE-2018216. Access on [2018-05-28]

[60] Yang S H, Liu X X, Chen B T, Li S X, Zheng Y J. CFD Models and Verification of the Downwash Airflow of an Eight-rotor UAV[C]//2019 ASABE Annual International Meeting. American Society of Agricultural and Biological Engineers, 2019: 1. doi: 10.13031/aim.201901018

[61] Zhang B, Tang Q, Chen L P, Xu M. Numerical simulation of wake vortices of crop spraying aircraft close to the ground. Biosystems Engineering, 2016, 145: 52-64. doi: 10.1016/j.biosystemseng.2016.02.014

[62] Liu C B, Song Z L. Research on the Impact of Meshing Density on the Efficiency of Computational Fluid Dynamics. Digital Manufacturing Science, 2019, 17(1): 54-58. (in Chinese with English abstract) doi: CNKI:SUN:ZZKX.0.2019-01-012 